\title{
Intrinsic apoptotic pathway activation increases response to anti-estrogens in luminal breast cancers
}

\author{
Michelle M. Williams', Linus Lee ${ }^{2}$, Thomas Werfel ${ }^{2}$, Meghan M. Morrison Joly ${ }^{1}$, Donna J. Hicks ${ }^{1}$, Bushra Rahman ${ }^{1}$, \\ David Elion', Courtney McKernan', Violeta Sanchez ${ }^{3}$, Monica V. Estrada ${ }^{3}$, Suleiman Massarweh ${ }^{4}$, Richard Elledge ${ }^{5}$, \\ Craig Duvall ${ }^{2}$ and Rebecca S. Cook ${ }^{1,2,6}$
}

\begin{abstract}
Estrogen receptor-a positive (ERa+) breast cancer accounts for approximately $70-80 \%$ of the nearly 25,0000 new cases of breast cancer diagnosed in the US each year. Endocrine-targeted therapies (those that block ERa activity) serve as the first line of treatment in most cases. Despite the proven benefit of endocrine therapies, however, ERa+ breast tumors can develop resistance to endocrine therapy, causing disease progression or relapse, particularly in the metastatic setting. Anti-apoptotic Bcl-2 family proteins enhance breast tumor cell survival, often promoting resistance to targeted therapies, including endocrine therapies. Herein, we investigated whether blockade of anti-apoptotic Bcl-2 family proteins could sensitize luminal breast cancers to anti-estrogen treatment. We used long-term estrogen deprivation (LTED) of human ERa+ breast cancer cell lines, an established model of sustained treatment with and acquired resistance to aromatase inhibitors (Als), in combination with BCl-2/BCl-xL inhibition (ABT-263), finding that ABT-263 induced only limited tumor cell killing in LTED-selected cells in culture and in vivo. Interestingly, expression and activity of the BCl-2-related factor Mcl-1 was increased in LTED cells. Genetic Mcl-1 ablation induced apoptosis in LTED-selected cells, and potently increased their sensitivity to ABT-263. Increased expression and activity of Mcl-1 was similarly seen in clinical breast tumor specimens treated with $\mathrm{Al}$ + the selective estrogen receptor downregulator fulvestrant. Delivery of Mcl-1 siRNA loaded into polymeric nanoparticles (MCL1 si-NPs) decreased Mcl-1 expression in LTED-selected and fulvestrant-treated cells, increasing tumor cell death and blocking tumor cell growth. These findings suggest that $\mathrm{Mcl}-1$ upregulation in response to anti-estrogen treatment enhances tumor cell survival, decreasing response to therapeutic treatments. Therefore, strategies blocking Mcl-1 expression or activity used in combination with endocrine therapies would enhance tumor cell death.
\end{abstract}

\section{Introduction}

The American Cancer Society estimated that approximately 25,0000 women were diagnosed with breast cancer in 2016 in the United States alone ${ }^{1}$. The most frequently

\footnotetext{
Correspondence: Rebecca S. Cook (Rebecca.cook@vanderbilt.edu)

'Department of Cancer Biology, Vanderbilt University School of Medicine, Nashville, TN, USA

${ }^{2}$ Department of Biomedical Engineering, Vanderbilt University School of Engineering, Nashville, TN, USA

Full list of author information is available at the end of the article

Michelle M. Williams and Linus Lee contributed equally to this work.

Edited by G. Giaccone.
}

diagnosed clinical breast cancers are those expressing estrogen receptor- $\alpha(E R \alpha)$, a nuclear receptor driving cell cycle progression. ER $\alpha+$ breast cancers are treated with targeted inhibitors that block $E R \alpha$ signaling, including selective ER $\alpha$ modulators (SERMS, e.g., tamoxifen), selective ER $\alpha$ downregulators (SERDs, e.g., fulvestrant) and AIs that decrease circulating estrogen in postmenopausal women. Although these treatments are successful for a large number of breast cancer patients, $15-30 \%$ display de novo or acquired resistance to 
anti-estrogens (reviewed in refs. ${ }^{2,3}$ ). Given the number of new diagnoses, and the numerous breast cancer-related deaths caused by anti-estrogen resistance each year, there is a need to identify molecular vulnerabilities in ER $\alpha+$ tumors for preventing or overcoming anti-estrogen resistance.

Resistance to many cancer treatments relies on evasion of cell death ${ }^{4}$, often caused by expression or activity of anti-apoptotic Bcl-2 family proteins (Bcl-A1, Bcl-2, Bcl$\mathrm{xL}, \mathrm{Bcl}-\mathrm{w}$, and $\mathrm{Mcl}-1)$. These factors prevent Bak/Bax oligomerization and pore formation in the outer mitochondrial membrane (as reviewed in refs. ${ }^{5,}$ ) by binding directly to Bak or $\mathrm{Bax}^{7}$, or to Bim, an activator of Bak/Bax oligomerization $^{8}$. ER $\alpha+$ breast cancers frequently overexpress anti-apoptotic Bcl-2, Bcl-xL, and $\mathrm{Mcl}-1^{9-12}$. Bcl-2 and $\mathrm{Bcl}-\mathrm{xL}$ are further elevated upon anti-estrogen treatment ${ }^{13-16}$, suggesting that $\mathrm{ER} \alpha+$ breast cancers may use anti-apoptotic Bcl-2 family members to drive cell survival and treatment resistance ${ }^{17,18}$.

Anti-estrogens are often cytostatic ${ }^{19}$, halting cell proliferation without activating apoptosis. Survival of tumor cells during treatment would increase the likelihood of recurrence upon treatment withdraw, and may enforce treatment resistance, suggesting that blockade of antiapoptotic $\mathrm{Bcl}-2$ proteins in combination with antiestrogens may decrease recurrence and/or resistance in $\mathrm{ER} \alpha+$ breast cancers. This idea has been tested using small molecular weight inhibitors known as 'BH3-mimetics,' designed to bind anti-apoptotic $\mathrm{Bcl}-2$ proteins within their $\mathrm{BH} 3$-interaction motif, preventing association with proapoptotic proteins Bax and $\mathrm{Bim}^{20}$. Although $\mathrm{Bcl}-2 / \mathrm{Bcl}-\mathrm{xL}$ inhibition using the BH3-mimetic ABT-737, or Bcl-2 specific inhibition, using the BH3-mimetic ABT-199, had little activity as single agents in breast cancers, their combination with tamoxifen resulted in tumor regression in some, but not all, patient-derived $\mathrm{ER} \alpha+$ breast cancer xenografts tested ${ }^{13}$, supporting a role for Bcl-2 in endocrine resistance. Other studies, however, show that $B C L 2$ is an ER $\alpha$ transcriptional target, and is decreased in tamoxifen-treated and tamoxifen-resistant xenografts ${ }^{21}$. These conflicting results require continued exploration of Bcl-2 family members ER $\alpha+$ breast cancers.

To investigate this, we used long-term estrogen deprivation (LTED) to model treatment with and acquired resistance to AIs in human luminal breast cancer cell lines. We found that Bcl-2/Bcl-xL inhibition did not increase cell death in LTED-selected cells. However, Mcl1 expression and activity were upregulated upon estrogen deprivation, as well as in response to fulvestrant. The recent development of Mcl-1-specific BH3-mimetics is allowing preclinical testing of Mcl-1 inhibition in some cancers $^{22-24}$, leading in some cases to clinical trials ${ }^{25}$. However, preclinical and clinical testing of Mcl-1 blockade in combination with endocrine inhibition in $E R \alpha+$ breast cancers is not fully explored. Targeted inhibition of Mcl-1 in ER $\alpha+$ breast cancers using Mcl-1 siRNA encapsulated in polymeric nanoparticles (si-NPs) increased tumor cell killing in LTED-selected cells and in fulvestrant-treated cells, demonstrating that antiestrogens prime ER $\alpha+$ cells for targeted Mcl-1 inhibition.

\section{Results}

Long-term estrogen deprivation does not sensitize cells to $\mathrm{Bcl}-2 / \mathrm{Bcl}-\mathrm{xL}$ inhibition

To model long-term treatment with AIs, three human $\mathrm{ER} \alpha+$ breast cancer cell lines, HCC1428, MCF7, and T47D, were cultured under estrogen deprivation for 3-6 months as described in previous studies ${ }^{26}$. While parental cells, which are maintained in estrogen-replete conditions, exhibited growth inhibition upon acute estrogen withdraw, LTED-selected cells grew at a similar or accelerated rate in estrogen-depleted media as compared to their growth in estrogen-replete conditions (Fig. 1a, b). Since previous studies show that antiapoptotic Bcl-2 family members Bcl-2 and Bcl-xL promote cell survival in tamoxifen-treated breast cancers ${ }^{13}$, we measured expression of $\mathrm{Bcl}-2$ and $\mathrm{Bcl}-\mathrm{xL}$ in parental and LTED-selected breast cancer cells, finding similar levels of Bcl-2 and Bcl-xL in LTED-selected HCC1428 and T47D cells as compared to their parental counterpoints cultured in estrogen-replete media (Fig. 1c). LTEDselected MCF7 cells expressed less Bcl-2 and less Bcl-xL as compared to parental counterparts. Interestingly, parental MCF7 cells upregulated Bcl-xL alone upon acute estrogen deprivation ( 3 days and 7 days), while parental HCC1428 cells upregulated Bcl-2 alone upon acute estrogen deprivation (Supplemental Figure S1A). These results suggest that, although acute estrogen deprivation may transiently enhance Bcl-2 and/or Bcl-xL, LTED does not.

Despite no obvious Bcl-2 and/or Bcl-xL protein upregulation in LTED-selected cells, it is possible that the antiapoptotic activities of Bcl-2 and/or Bcl-xL (i.e. sequestration of pro-apoptotic Bim or Bax) support cell survival and therapeutic resistance in this setting. To test this, activity of Bcl-2 and Bcl-xL was blocked in LTED-selected

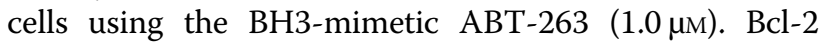
immuno-precipitates assessed by western analysis for Bim confirmed that ABT-263 reduced $\mathrm{Bcl}-2 / \mathrm{Bim}$ interactions (Fig. 1d and Supplemental Figure S1B). Previous reports show that ABT-263 treatment of HCC1428, MCF7, and T47D cells induced caspase-3/7 activity after $4 \mathrm{~h}^{12}$, similar to observations in other cell lines ${ }^{27}$. In contrast, ABT-263 did not increased caspase-3/7 activity in MCF7-LTED and T47D-LTED cells at 4 or $24 \mathrm{~h}$ (Fig. 1e), while HCC1428LTED cells responded to ABT-263 with a subtle increase in caspase-3/7 activity at $4 \mathrm{~h}$ only. These results were confirmed in vivo using MCF7-LTED xenografts treated 


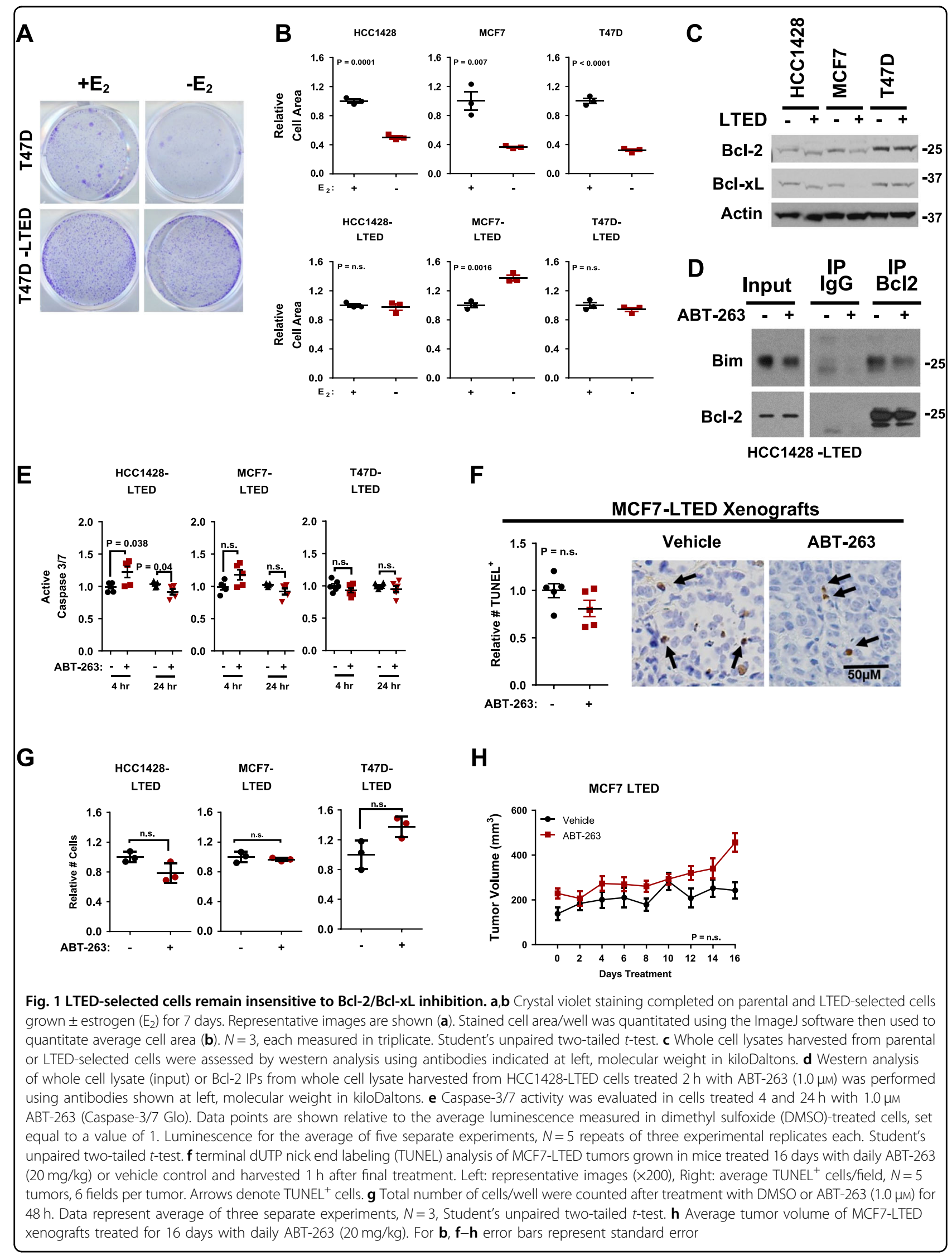


daily with ABT-263 $(20 \mathrm{mg} / \mathrm{kg})$, which did not increase tumor cell death as assessed by terminal dUTP nick end labeling (TUNEL) analysis (Fig. 1f). Consistent with these results, cell growth (total cell number) of LTED-selected cells was not affected by $48 \mathrm{~h}$ treatment with ABT-263 (Fig. 1g). Similarly, MCF7-LTED xenograft growth (tumor volume) was not affected by daily ABT-263 treatment (Fig. 1h), even though Bim/Bcl-2 interactions were disrupted by ABT-263 (Supplemental Fig. 2a). Tumor cell proliferation also was unaffected by ABT-263 (Supplemental Fig. 2b). These data suggest that LTED, such what is achieved by AIs, may not prime ER $\alpha+$ breast cancer cells for Bcl-2/Bcl-xL targeting.

\section{Mcl-1 expression increases under long-term estrogen deprivation}

Previous studies demonstrated that $\mathrm{Mcl}-1$, a related anti-apoptotic Bcl-2 protein, is upregulated upon Bcl-2/ $\mathrm{Bcl}-\mathrm{xL}$ inhibition, resulting in resistance to ABT-263 ${ }^{22,28-32}$. In fact, Mcl-1 expression levels correlated inversely with sensitivity to ABT-263 in untreated $\mathrm{ER} \alpha+$ breast cancer cells ${ }^{12}$. Interestingly, Mcl-1 levels were elevated in HCC1428-LTED, MCF7-LTED, and T47D-LTED cells as compared to parental counterparts (Fig. 2a). We also measured relative BIK and PUMA transcript levels in cells selected for LTED resistance, based on previous reports that anti-estrogen treatments result in upregulation of Bik, but not Puma ${ }^{33,}{ }^{34}$. Similar to previous reports, we found increased BIK in MCF7LTED and T47D-LTED over what was seen in MCF7 parental and T47D parental cell lines, respectively, while PUMA transcript levels were unaltered in LTED cells as compared to parental counterparts (Supplemental Figure 3a). Although the trend towards $B I K$ upregulation in HCC1428-LTED cells over parental HCC1428 cells did not reach statistical significance, together these findings suggest that $\mathrm{ER} \alpha+$ breast cancer cells respond to sustained estrogen deprivation through $B I K$ and Mcl-1 upregulation. $^{33,} 34 \mathrm{Mcl}-1$ upregulation upon sustained estrogen deprivation was confirmed by immunohistochemical staining in MCF7 xenografts grown in ovariectomized hosts (thus, estrogen deprived for 42 days), revealing increased $\mathrm{Mcl}-1$ staining intensity, as compared to MCF7 xenografts grown in ovariectomized hosts supplemented with 42-day release estrogen pellets (Fig. 2b, and Supplemental Figure S3b). We used Proximity Ligation Assay $\left(\mathrm{PLA}^{35}\right)$, a technique measuring protein-protein interactions in situ, to quantitate $\mathrm{Mcl}-1$ activity (Mcl$1 /$ Bim interactions) in MCF7 xenografts. Fluorescent puncta, indicating $\mathrm{Bim} / \mathrm{Mcl}-1$ interaction, were increased in tumors grown in ovariectomized mice without estrogen supplementation (Fig. 2c), suggesting that, despite upregulation of pro-apoptotic forces such as $B I K$, increased Mcl-1 expression and activity upon sustained estrogen deprivation also increase, perhaps counteracting $B I K$ upregulation and promoting cell survival.

\section{Fulvestrant primes luminal breast cancers for $\mathrm{Mcl}-1$ targeting}

We next examined Bcl-2, Bcl-xL, and Mcl-1 expression changes in response to fulvestrant, a SERD that blocks estrogen binding to $\mathrm{ER} \alpha$ and induces $\mathrm{ER} \alpha$ degradation. Parental HCC1428, MCF7, and T47D cells treated $24 \mathrm{~h}$ with fulvestrant $(1.0 \mu \mathrm{M})$ showed decreased $B C L 2$ transcripts (Fig. 3a), while BCL2L1 (Bcl-xL) levels increased in HCC1428 and T47D cells. MCL1 transcripts were elevated in all three fulvestrant-treated cell lines. Mcl-1 protein expression in parental MCF7 and T47D cells increased upon fulvestrant treatment, while Bcl-2, detected at very low levels in MCF7 and T47D cells, diminished further in response to fulvestrant (Fig. 3b), as did Bcl-xL. Importantly, fulvestrant did not induce significant levels of caspase-3/7 activity in cultured HCC1428, MCF7, or T47D cells (Fig. 3c). These findings were verified in vivo using MCF7 and T47D xenografts treated 7 days with fulvestrant, revealing fulvestrantmediated ER $\alpha$ downregulation (Supplemental Figure S4) and Mcl-1 upregulation (Fig. 3e). Further, PLA-mediated detection of $\mathrm{Mcl}-1 / \mathrm{Bim}$ interactions demonstrated that fulvestrant increased Mcl-1 activity (Fig. 3d). Bcl-2 levels increased in fulvestrant-treated MCF7 (but not T47D) tumors, while $\mathrm{Bcl}-\mathrm{xL}$ showed relatively little change (Fig. 3d).

\section{Genetic targeting of MCL1 increases apoptosis in LTED-selected breast cancer cells}

We delivered siRNA sequences directed against Mcl-1 to LTED-selected ER $\alpha+$ breast cancer cells as an experimental approach at targeted and highly selective Mcl-1 inhibition. As a control, we used luciferase-directed siRNA sequences. Delivery of siRNA sequences was achieved using polymeric siRNA nanoparticles (si-NPs), including MCL1 si-NPs and LUC si-NPs, respectively. These recently developed nanocarriers enable effective siRNA delivery/activity in cultured cells due to high cell uptake and efficient endosomal escape. Also, unlike other off-the-shelf agents, these NPs can be directly translated into future in vivo studies due to sufficient serum stability that enables i.v. injection and biodistribution/bioactivity within breast tumors ${ }^{36}$. Cells were assessed for Mcl-1 knockdown by western analysis $48 \mathrm{~h}$ after treatment with si-NPs, demonstrating that MCL1 si-NPs decreased Mcl-1 protein expression in all three LTED cells (Fig. 4a). Importantly, Bcl-2 and Bcl-xL levels remained unchanged despite Mcl-1 knockdown in MCL1 si-NP-treated cells, confirming target sequence specificity, and ruling out acute compensatory upregulation of $\mathrm{Bcl}-2$ and $\mathrm{Bcl}-\mathrm{xL}$ upon Mcl-1 gene targeting. 


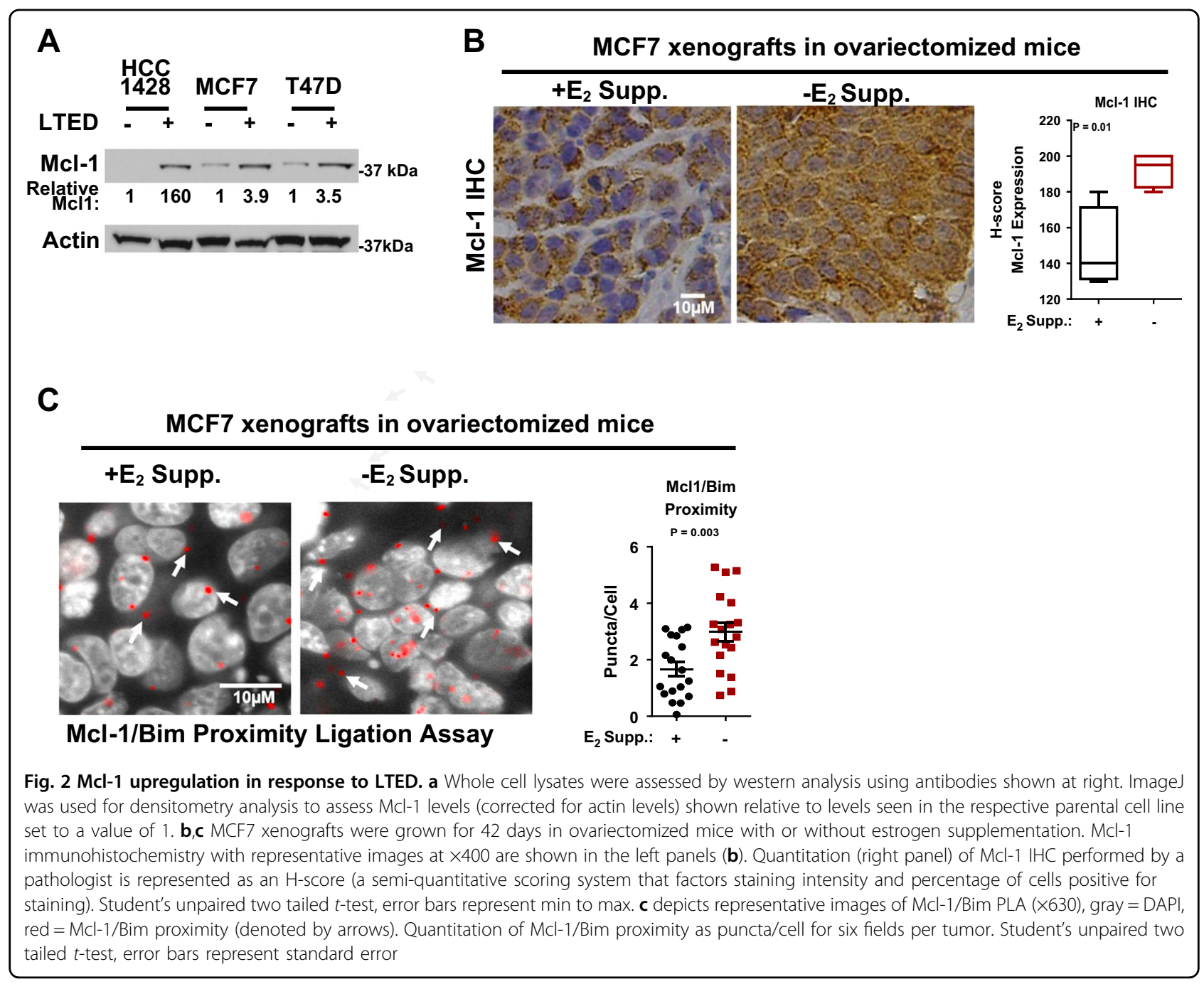

HCC1428-LTED, MCF7-LTED, and T47D-LTED cells treated with MCL1 si-NPs displayed increased caspase-3/ 7 activity as compared to cells treated with LUC si-NPs (Fig. 4b). Caspase-3/7 activity was increased further in LTED-selected cells when MCL1 si-NPs were used in combination with ABT-263. MCL1 si-NPs decreased growth of LTED-selected cells as compared to LUC siNPs (Fig. 4c), and increased growth inhibition in response to ABT-263.

\section{Genetic targeting of MCL1 increases apoptosis in Fulvestrant-treated breast cancer cells}

We next examined the impact of MCL1 si-NPs in parental $\mathrm{ER} \alpha+$ breast cancer treated with fulvestrant, first verifying Mcl-1 knockdown in parental HCC1428, MCF7, and T47D cells at $48 \mathrm{~h}$ post-treatment, revealing 70-90\% fewer MCL1 transcripts as compared to cells treated with LUC si-NPs (Fig. 5a). These results were confirmed by western analysis at this same timepoint (Fig. 5b). We next measured caspase-3/7 activity in ER $\alpha+$ breast cancer cells $48 \mathrm{~h}$ after treatment with MCL1 si-NPs. Unlike what was seen in LTED-selected cells, which exhibited increased cell death upon knockdown of Mcl-1, two of three parental cells did not increase caspase-3/7 activity in response to MCL1 si-NPs (Fig. 5c). Consistent with previous reports, fulvestrant as a single agent also did not induce caspase-3/7 activity in HCC1428, MCF7, or T47D cells. However, the MCL1 si-NPs combined with fulvestrant robustly increased caspase-3/7 activity in HCC1428, MCF7, or T47D cells. Interestingly, MCL1 si-NPs alone decreased growth of parental MCF7, but not HCC1428 or T47D cells, over 7 days (Fig. 5d). As expected, fulvestrant decreased growth of ER $\alpha+$ cell lines. However, the combination of fulvestrant + MCL1 si-NPs decreased growth of HCC1428, MCF7, and T47D to a greater extent than either agent alone. 


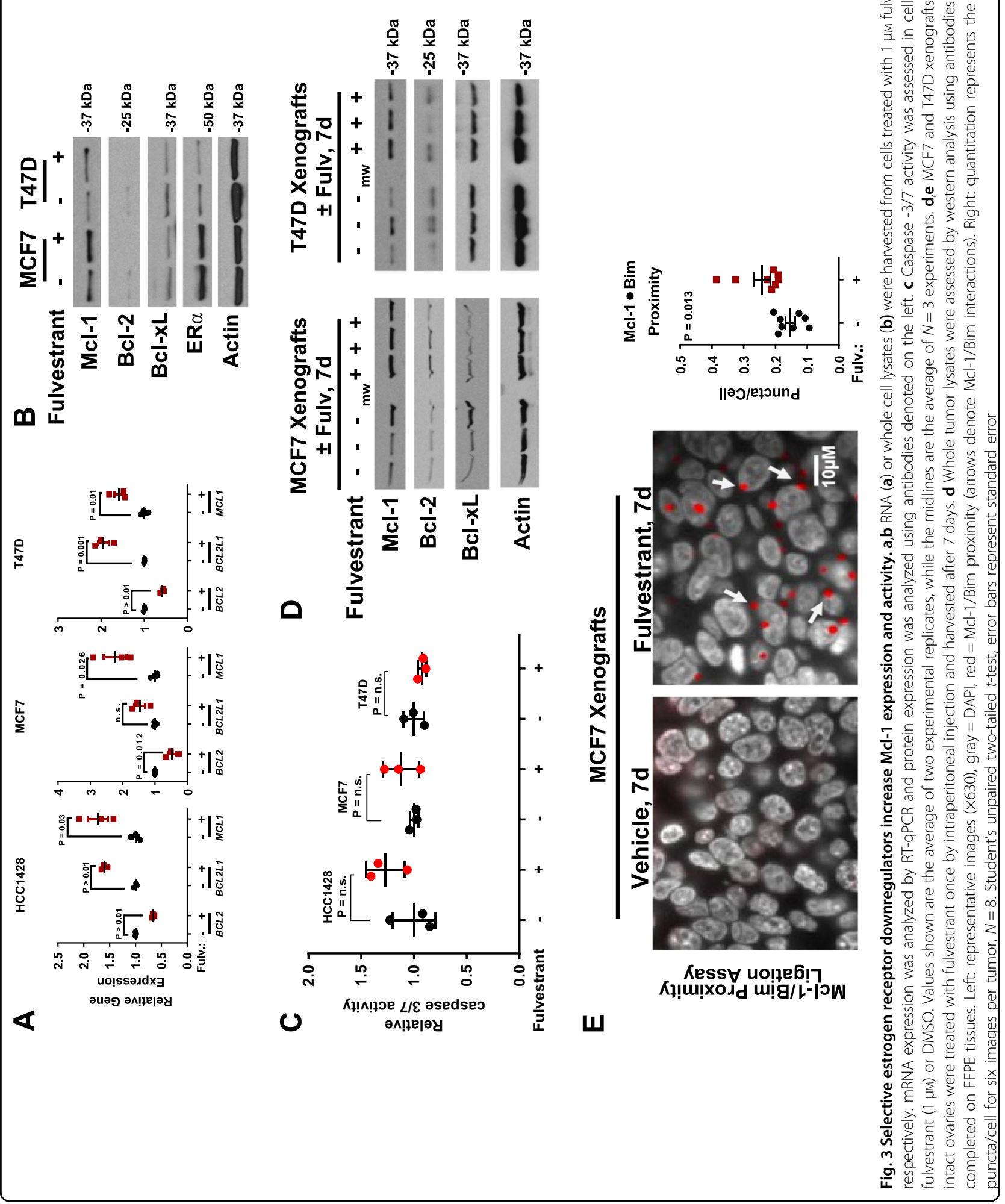




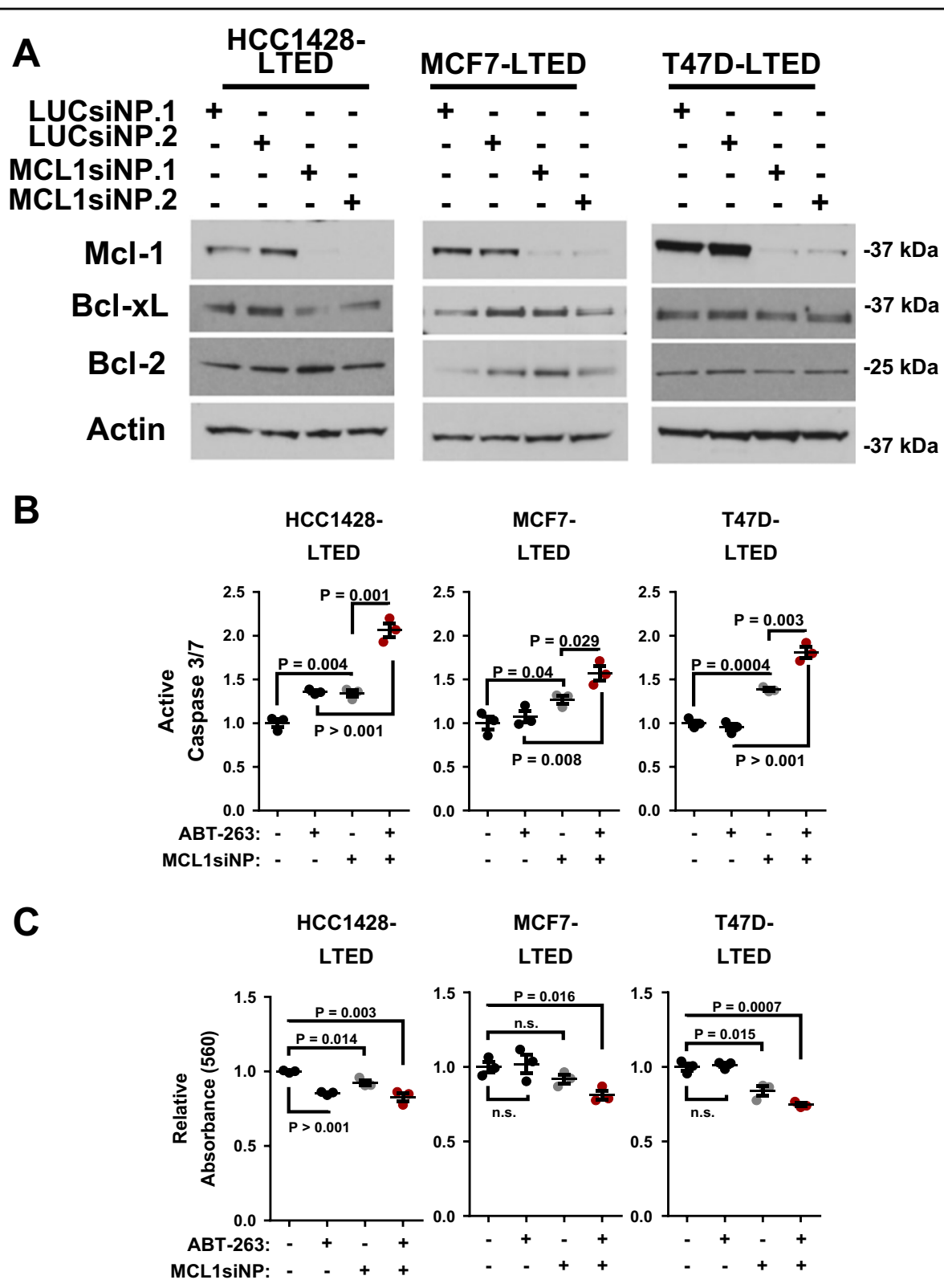

Fig. 4 Mcl-1 inhibition restores sensitivity of LTED breast cancers to ABT-263. a Whole cell lysates from LTED-selected cells were assessed by western analysis for antibodies indicated at left after treatment with siNPs loaded with $50 \mathrm{~nm}$ siControl or siMcl 1 for $48 \mathrm{~h}$. $\mathbf{b}, \mathbf{c}$ LTED-selected cells were treated for $48 \mathrm{~h}$ with $50 \mathrm{~nm}$ si-NP. $1.0 \mu \mathrm{m}$ ABT-263 was added for the final $4 \mathrm{~h}$. Caspase-3/7 activity was measured (Caspase-3/7 Glo, b) and used to calculate average luminescence, relative to values obtained for LUC si-NP-treated cells with DMSO treatment, set to a value of 1. $N=3$, each assessed in duplicate. Student's unpaired two-tailed t-test, error bars represents standard error. Total protein was quantitated using an SRB assay (c), relative to values obtained for LUC si-NP-treated cells with DMSO treatment, set to a value of 1. N=3, each assessed in duplicate. Student's unpaired two-tailed $t$-test, error bars represent standard error

Mcl-1 activity increases clinically, highlighting a molecular vulnerability

To assess whether Mcl-1 is an essential tumor cell survival factor during a standard of care treatment regimen (i.e. first-line treatment with AIs, followed by second-line treatment with fulvestrant upon AI resistance), we first analyzed matched pairs of clinical breast tumor specimens from patients with $\mathrm{ER} \alpha+$ breast cancers for Mcl-1/Bim interactions by PLA. Breast tumor biopsies were collected from post-menopausal women with a new diagnosis of $E R \alpha+$ breast cancer as part of a single-stage, single institution phase II neoadjuvant trial ${ }^{37,38}$. Tumors were biopsied $24 \mathrm{~h}$ prior to any treatment (day 0 ). Patients were treated with a single fulvestrant dose (Faslodex, 250 


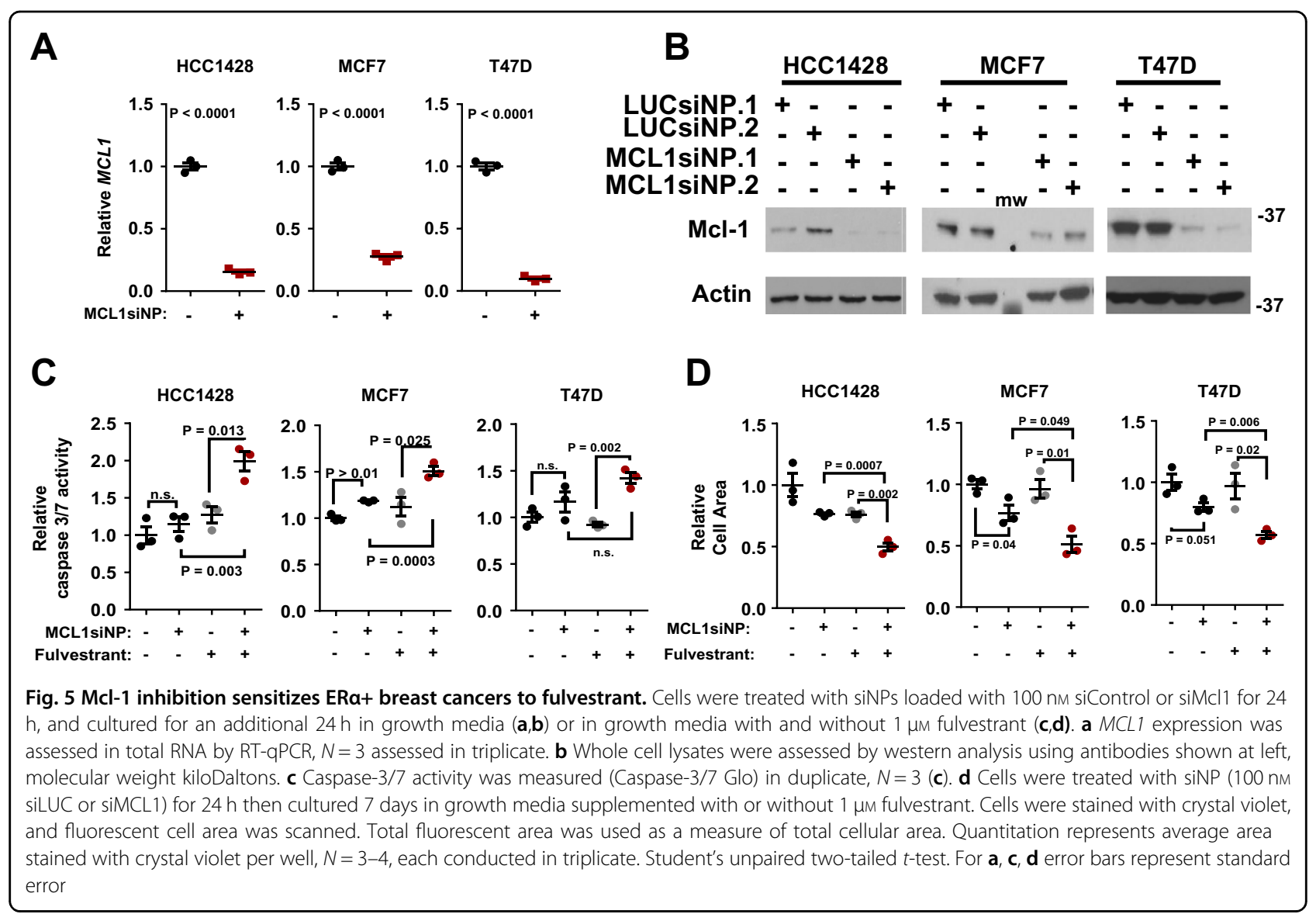

mg) followed by daily treatment with the AI anastrozole (Arimidex, $1 \mathrm{mg}$ daily). In some cases, patients also received daily treatment with the epidermal growth factor receptor (EGFR) inhibitor gefitinib. Tumor biopsies were collected again on treatment day 21. These studies revealed that Bim interactions with $\mathrm{Mcl}-1$ were increased in post-treatment specimens (day 21) as compared to those collected prior to treatment (day 0, Fig. 6a), consistent with the results shown here in animal models. Although the inclusions of the EGFR kinase inhibitor gefitinib is a caveat of the clinical data set that is inconsistent with the endocrine treatment modalities used herein, these results support the idea that increased Mcl-1 expression and activity may be clinically meaningful.

HCC1428-LTED, MCF7-LTED, and T47D-LTED cells were treated with MCL1 si-NPs (or LUC si-NPs, as a control), in the presence or absence of fulvestrant to assess the impact of Mcl-1 in treatment response (or resistance) to the second-line anti-estrogen fulvestrant in cells already resistant to estrogen deprivation. Similar to what was seen in parental HCC1428 and MCF7 cells, Mcl-1 protein expression was upregulated in HCC1428LTED and MCF-LTED in response to fulvestrant (Fig. 6b). However, MCL1 si-NPs resulted in nearly complete knockdown of Mcl-1 protein expression, negating the ability of cells to upregulate Mcl-1 in response to fulvestrant. As previously reported by other groups, HCC1428-LTED, MCF7-LTED, and T47D-LTED retain their sensitivity to fulvestrant, as demonstrated by modestly increased caspase-3/7 activity (Fig. 6c) and substantially reduced cell growth in monolayer colony assays (Fig. 6d). However, MCL1 si-NPs used in combination with fulvestrant increased caspase-3/7 activity to a greater extent than fulvestrant alone (Fig. 6c) and decreased colony formation to a greater extent than fulvestrant alone (Fig. 6d), confirming that LTED cells, which already use Mcl-1 upregulation to support cell survival, can further increase Mcl-1 in response to additional pressure placed on the ER $\alpha$ pathway by fulvestrant, thus reinforcing their reliance upon Mcl-1 for cell survi$\mathrm{val}^{39}$. These findings support the feasibility of therapeutic Mcl-1 gene ablation in combination with anti-estrogens ${ }^{39}$ (Fig. 6e).

\section{Discussion}

Previous preclinical studies show that anti-apoptotic Bcl-2 family proteins promote resistance to standard of care breast cancer therapies, including endocrine 


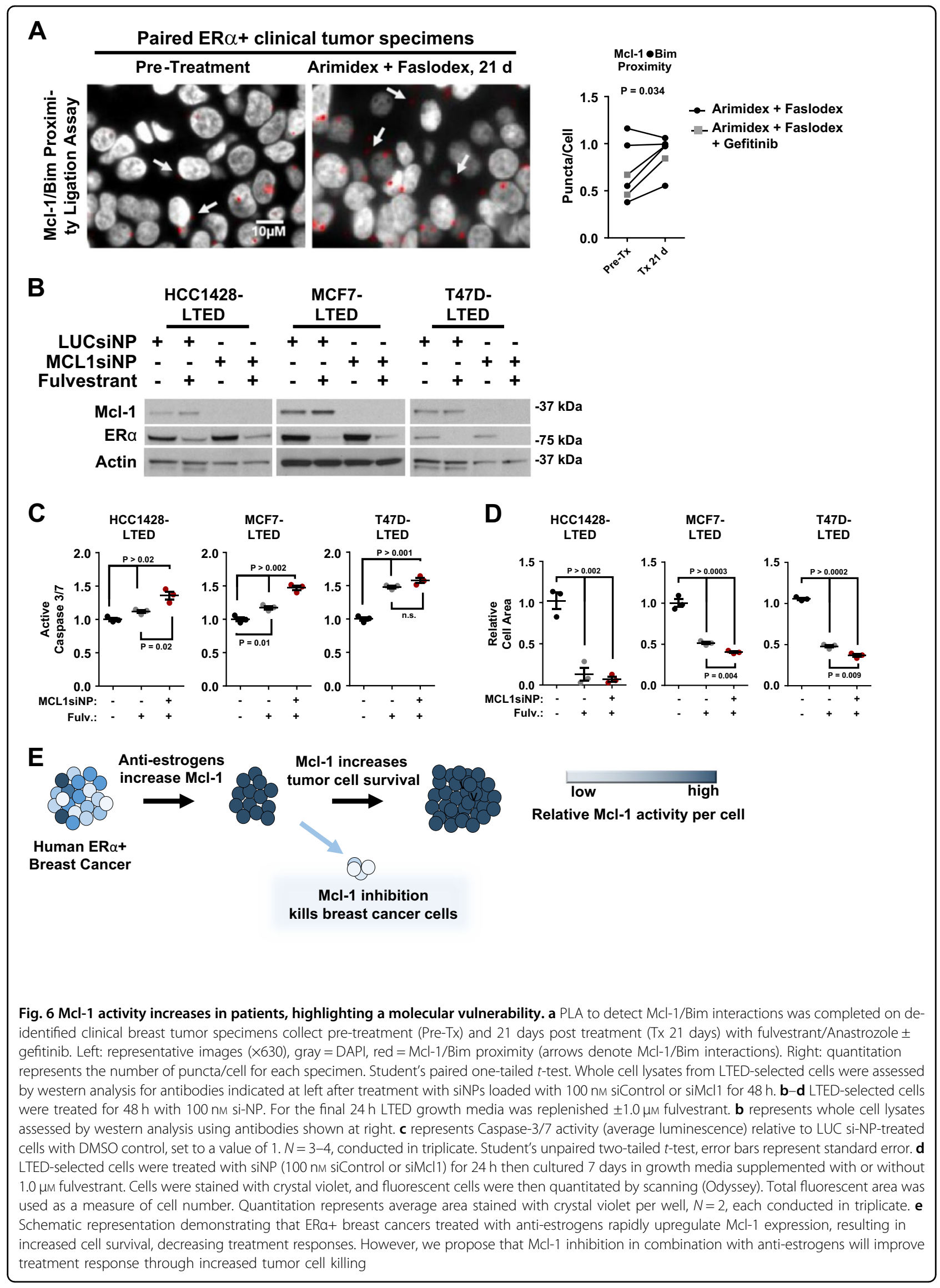


inhibitors $^{13-16}$, HER2 inhibitors ${ }^{40}$, and chemotherapies ${ }^{41-43}$. An impactful study using preclinical models of $\mathrm{ER} \alpha+$ breast cancers showed extreme sensitive to the combination of tamoxifen and the $\mathrm{Bcl}-2 / \mathrm{Bcl}-\mathrm{xL}$ inhibitor $\mathrm{ABT}$ 737 or the Bcl-2 specific inhibitor ABT-199, suggesting that Bcl-2 drives resistance of $\mathrm{ER} \alpha+$ breast cancers to tamoxifen ${ }^{13}$. Based on these and other studies, clinical testing of ABT-199 in combination with tamoxifen has begun $^{44}$. However, in a separate study, Bcl-2 expression was decreased in MCF7 xenografts upon tamoxifen treatment and decreased further upon acquisition of tamoxifen resistance ${ }^{21}$, consistent with the clinical finding that $\mathrm{Bcl}-2$ expression often predicts a favorable prognosis $^{45}$, and with the idea that Bcl-2 is a transcriptional target of ER $\alpha$. These conflicting reports, in addition to a lack of studies observing the role of other anti-apoptotic factors in ER $\alpha+$ breast cancer resistance, led us to further explore the role of Bcl-2, as well as Bcl-xL and Mcl-1, in response of $E R \alpha+$ breast cancers to endocrine inhibition.

We found that LTED cells were not sensitive to a physiologically relevant dose of ABT-263, a Bcl-2/Bcl-xL inhibitor that functions similarly to ABT-737. ABT-263 induced tumor cell killing transiently in only one of three LTED-selected ER $\alpha+$ breast cancer cell lines tested, but this response was not sustained, and ABT-263 did not decrease tumor cell growth in culture or in vivo (Fig. 1e-h). Interestingly, short-term estrogen deprivation (0-48 h) increased Bcl-2 and/or Bcl-xL levels in some, but not all, ER $\alpha+$ cell lines (Supplemental Figure S1A), although sustained estrogen deprivation caused $\mathrm{Bcl}-2$ and Bcl-xL levels to decrease substantially (Fig. 1c). It is possible that short-term treatment $(0-48 \mathrm{~h})$ with tamoxifen or AIs increases Bcl-2 and/or Bcl-xL levels, priming ER $\alpha+$ breast tumor cells for Bcl-2/Bcl-xL inhibition, as was seen in $\mathrm{ER} \alpha+$ patient-derived xenograft models ${ }^{13}$. However, upon acquisition of resistance, $\mathrm{Bcl}-2 / \mathrm{Bcl}-\mathrm{xL}$ levels become depleted, and survival then depends on different antiapoptotic factors. These ideas will require further exploration in future studies.

Transient sensitivity to ABT-263, as seen in HCC1428LTED cells, was previously explored in ER $\alpha+$ breast cancer cell lines grown in estrogen replete conditions ${ }^{46}$. These studies showed that Mcl-1, a Bcl-2-related antiapoptotic factor, is a key driver of ABT-263 resistance, supporting the concept of compensatory and overlapping functions across anti-apoptotic $\mathrm{Bcl}-2$ family members. Therefore, we were motivated to explore the role of Mcl-1 in our LTED models that were not only resistant to estrogen deprivation, but also to ABT-263. We found abundant Mcl-1 expression (but not $\mathrm{Bcl}-2$ or $\mathrm{Bcl}-\mathrm{xL}$ ) upon LTED in cell culture and in vivo (Figs. 1c and 2a,b), suggesting that Mcl-1, rather than Bcl-2 or Bcl-xL, may be the primary survival factor in AI-resistant $\mathrm{ER} \alpha+$ breast cancers.
Previous reports demonstrate that fulvestrant treatment of $E R \alpha+$ breast cancer cells causes mRNA upregulation of the pro-apoptotic BH3-only family member, $B I K$, but not other BH3-only factors, such as PUMA ${ }^{33,}{ }^{34}$. Consistent with these reports, we found that fulvestrant-treated HCC1428, MCF7, and T47D cells also upregulated BIK mRNA, while PUMA levels remained unchanged (Supplemental Figure S5). Despite increased expression of $B I K$, fulvestrant treatment of HCC1428, MCF7, and T47D cells resulted in only modest induction of caspase-3/7 activity in HCC1428 and MCF7 cells, and did not impact caspase-3/7 activity in T47D cells (Fig. 3c) ${ }^{14}$. Interestingly, we found increased Mcl-1/Bim interactions in fulvestranttreated MCF7 xenografts (Fig. 3d,e), and in clinical ER $\alpha+$ tumor specimens treated with Faslodex/Arimidex (Fig. 6a). These findings support previous reports showing increased $B I K$ expression upon short-term estrogen deprivation and acute fulvestrant treatment ${ }^{33,}{ }^{34}$, demonstrating that Bik mediates endocrine inhibitorinduced apoptosis. However, our data suggest that upon treatment resistance increased pro-apoptotic signals are thwarted by Mcl-1 upregulation, thus promoting tumor cell survival.

Although previous reports demonstrate that Mcl-1 is a transcriptional target of $\mathrm{ER} \alpha^{47}$, data presented herein show that multiple endocrine inhibitors increase Mcl-1 expression and activity in cell culture, in vivo, and in clinical samples, suggesting that ER $\alpha$ may not be a dominant transcriptional activator of Mcl-1. One possibility is that ER $\alpha$ binding sites within the Mcl-1 promoter may repress Mcl-1 gene expression, although this hypothesis has not been tested. Alternatively, the process of Mcl-1 regulation may be very complex, such that ER $\alpha$ inhibition using estrogen deprivation or fulvestrant may change other ER $\alpha$-regulated transcriptional programs that influence Mcl-1 gene regulation in an indirect manner. These interesting hypotheses will require further investigation, as $M C L 1$ gene regulation is likely a key deciding element governing tumor cell death in therapeutically treated ER $\alpha+$ breast cancers.

Since high Mcl-1 activity is known to promote resistance to ABT-263 in breast cancers ${ }^{12,32,48}$, these findings suggest that LTED-mediated Mcl-1 induction may indirectly increase resistance to $\mathrm{Bcl}-2 / \mathrm{Bcl}-\mathrm{xL}$ inhibition. Thus, it became imperative to inhibit Mcl-1 in combination with $\mathrm{Bcl}-2 / \mathrm{Bcl}-\mathrm{xL}$ in LTED cell lines. Mcl-1 inhibition was achieved using nanoparticles loaded with Mcl-1 siRNA (MCL1 si-NPs). The combination of ABT-263 with MCL1 si-NPs increased tumor cell killing and diminished tumor cell growth in each LTED cell line tested (Fig. 4b,c). These findings support the idea that inhibition of multiple anti-apoptotic Bcl-2 family proteins may be required to maximize tumor cell killing. However, a pan-Bcl-2 family inhibitor would have to overcome the 
challenges of clinical toxicities. For instance, ABT-263 was previously suspended from clinical trials due to extreme thrombocytopenia ${ }^{49},{ }^{50}$. Further, it is possible that Mcl-1 targeting alone will be toxic, because Mcl-1 is essential to the survival of normal tissues, such as the heart $^{51}$, brain $^{52,53}$, and the immune compartment ${ }^{54-56}$. For this reason, we explored Mcl-1 targeting using siRNA, which offers a genetically selective, and possibly less toxic, method to deplete Mcl-1. The nanoparticles into which siRNA sequences were loaded have been vetted for toxicity, and are optimized for superior systemic in vivo delivery over what can be achieved using commercially available reagents. Further, si-NPs penetrate less into healthy tissues as compared to small molecule inhibitors, thus increasing tumor-specific delivery via the enhanced permeability and retention effect ${ }^{22,} 23,57$, a term describing the leaky vasculature of tumors caused by rapid and irregular tumor angiogenesis. Based on their relative size, si-NPs passively enter and accumulate in tumors ${ }^{58}$, limiting extra-tumoral side effects. Due to these favorable pharmacokinetics, many nanoparticle-based drugs, including siRNA-loaded particles, are in various stages of clinical development [as reviewed in ref. ${ }^{59}$ ].

Although si-NPs targeting Mcl-1 remain to be tested in vivo, Mcl-1-specific inhibition in cell culture-based models dramatically reduced Mcl-1 expression in both LTED and parental breast cancer cells (Figs. 4a and 5b), increasing tumor cell killing in cells that have developed resistance to current standard of care therapies. Further, we tested the utility of MCL1 si-NPs in combination with fulvestrant, because Mcl-1 expression was induced upon short-term fulvestrant treatment in culture and in vivo (Fig. 3b, d), and tumor cell death was increased upon combined treatment with fulvestrant and MCL1 si-NPs (Fig. 5c). Additionally, fulvestrant treatment further increased tumor cell killing induced by MCL1 si-NPs in LTED-selected cells lines (Fig. 6c), suggesting that Mcl-1 targeting in combination with second-line fulvestrant treatment may be a clinical strategy to increase tumor cell death in ER $\alpha+$ breast cancer patients. Overall, these studies demonstrate $\mathrm{ER} \alpha+$ breast cancers treated with standard of care endocrine inhibitors may induce Mcl-1 expression and/or activity, which could represent a potential dynamic biomarker of sensitivity to Mcl-1 inhibition. Further, inhibition of Mcl-1 in tumors that are primed by endocrine inhibition may be viable strategy to increase tumor cell killing and breast cancer patient survival.

\section{Materials and methods}

\section{Cell culture}

All cells lines were purchased from the American Tissue Type Collection (Homo sapiens ATCC CRL 2327; HTB22; HTB-133). Cells were maintained in normal growth media (DMEM - Dulbecco's Modified Eagle Medium, 10\% fetal bovine serum, $1 \times$ antibiotics/anti-mycotics). To generate LTED cells, each cell line was cultured in LTED growth media (phenol-red free Opti-Mem, 10\% charcoal stripped fetal bovine serum, $1 \times$ antibiotics/anti-mycotics) until they grew at a similar rate to parental cells, 3-6 months. Growth in monolayer (crystal violet) and cell count analyses were completed as in ref. ${ }^{12}$. In cases where parental cell lines were treated with or without estrogen, estrogen was withdrawn after cells were seeded for the corresponding experiment and were cultured in LTED growth media supplemented with or without $2.0 \mathrm{~nm} 17 \beta-$ estradiol (Sigma). Otherwise, parental cells were grown and maintained in the presence of estrogen. Fulvestrant (Selleckchem) and ABT-263 (Selleckchem) were dissolved in dimethyl sulfoxide (DMSO) and used at a final concentration of $1.0 \mu \mathrm{M}$.

\section{Caspase-3/7 activity}

Five thousand cells/well were plated in 96-well plates in growth media or LTED growth media, and treated with $1.0 \mu \mathrm{M}$ ABT-263. For experiments with si-NPs, 5000 cells/ well (parental cells) or 10,000 cells/well (LTED-selected cells) were plated in 96-well plates and treated with si-NPs for $24 \mathrm{~h}$ when growth media was replenished $\pm 1.0 \mu \mathrm{M}$ fulvestrant. Caspase activity was determined following the Capsase 3/7-Glo assay (Promega) according to the manufacturer's instructions at $4 \mathrm{~h}$ after adding ABT-263 or $48 \mathrm{~h}$ after adding si-NPs. Overall luminescence was read on a Glomax Mutli+ Detection System luminometer (Promega).

\section{Immunoprecipitation and western analysis}

Ten micrograms Bcl-2 (DAKO) or IgG control (Santa Cruz Biotechnologies) antibodies were crosslinked to 50 $\mu \mathrm{L}$ protein A/G agarose beads (Santa Cruz Biotechnologies) using $5 \mathrm{~mm}$ BS3 (Sigma-Aldrich) by rotating at room temperature for $30 \mathrm{~min}$. BS3 was quenched using $15 \mu \mathrm{L}$ $1.0 \mathrm{M}$ Tris ( $\mathrm{pH} 7.4)$, and beads were washed $3 \times$ with onefourth Nonident-P40 (NP-40) lysis bufferNLB (see below) diluted in $1 \times$ PBS. $10 \mu \mathrm{L}$ crosslinked beads (5 $\mu$ g antibody) were rotated overnight at $4{ }^{\circ} \mathrm{C}$ with $1000 \mu$ g protein lysate, harvested in $0.1 \%$ Nonident-P40 (NP-40) lysis buffer (NLB) supplemented with protease inhibitor cocktail (Roche), proteasome inhibitor MG-132 (Selleckchem), and sodium orthovanadate (Pierce) as described in ref. ${ }^{12}$. Samples were washed with NLB, boiled in $1 \times$ reducing sample buffer (NuPAGE, Invitrogen) and run on a 4-12\% sodium dodecyl sulfate polyacrylamide (SDS-PAGE) gel as detailed in ref. ${ }^{12}$. Proteins were transferred to nitrocellulose (iBlot, Invitrogen), blocked 3\% gelatin in Tris buffered saline, 0.1\% Tween-20 (Sigma-Aldrich), and 3\% cold fish gelatin (Sigma-Aldrich), then probed with antibodies against Bim (Cell Signaling Technology, 1:500), 
Mcl-1 (Santa Cruz Biotechnologies, 1:500), Bcl-xL (Santa Cruz Biotechnologies, 1:500), Actin (Cell Signaling, 1:10,000) and Bcl-2 (DAKO, 1:1000).

\section{Mice}

Mice were housed under pathogen-free conditions and all experiments were in accordance with AAALAC guidelines and with Vanderbilt University Institutional Animal Care and Use Committee approval. MCF7 and MCF7-LTED xenografts were generated as described previously $^{60}$. Tumor volume was measured every other day once tumors became palpable. Treatment of mice began when tumors reached $200 \mathrm{~mm}^{3}$. Mice were randomized and treated with $50 \mu \mathrm{L}$ vehicle control $(0.1 \%$ Tween 80 and $0.5 \%$ methylcellulose) or ABT-263 (20 mg/ $\mathrm{kg}$, dissolved in vehicle control) daily by gavage for 16 days or until tumor volume reached $1000 \mathrm{~mm}^{3}$. Tissues were collected $1 \mathrm{~h}$ after final treatment. MCF7 xenografts were similarly generated and treated with fulvestrant as described in ref. ${ }^{60}$.

\section{Histological analyses}

Tumors were fixed in 10\% formalin. Formalin-fixed, paraffin-embedded tumor sections were assessed by terminal dUTP nick end labeling (TUNEL) analysis using the In Situ TUNEL detection kit (source) and for Mcl-1 using methods described in ref. ${ }^{41}$ and anti-Mcl-1 antibody (Santa Cruz Biotechnologies).

\section{Proximity ligation assay}

Formalin-fixed paraffin-embedded sections of human breast tumor biopsies collected as part of a single-stage, single institution phase II neoadjuvant trial, in which post-menopausal women with newly diagnosed breast cancer were treated with anastrozole (1 mg daily, Arimidex) and fulvestrant (250 mg, once per month, Faslodex). After a baseline tumor core biopsy on day 0, patients received treatment (Faslodex and Arimidex). In some cases, patients received the epidermal growth factor receptor (EGFR) kinase inhibitor gefitinib (Iressa) ${ }^{37,}{ }^{38}$. Tissues were rehydrated using ethanol. Following antigen retrieval using citric acid, the Duolink (Sigma) PLA kit was used according to the manufacturer's directions using Mcl-1 (Santa Cruz Biotechnologies, 1:25) and Bim (Santa Cruz Biotechnologies, 1:25) antibodies. A Duolink DAPI mounting media was used to counter stain cells. Images were taken at $63 \times$ and total puncta/nuclei were counted per field using the ImageJ (Windows 64) Software.

\section{siRNA-loaded nanoparticles}

All polymers were synthesized and characterized as described previously ${ }^{36}$. Two polymers (p(DMAEMA-coBMA), DB) and (PEG-b-p(BMA-co-DMAEMA), PDB) were used to form si-NPs. Both polymers were dissolved in a $10 \mathrm{~mm}$ citric acid buffer ( $\mathrm{pH} 4.0)$. The siRNA (50 $\mu \mathrm{M}$ in $\mathrm{diH}_{2} \mathrm{O}$ ) was initially complexed at 4:1 N:P ratio (ratio of polymer amines:siRNA backbone phosphates) with the DB polymer $(0.5 \mathrm{mg} / \mathrm{mL}$ polymer concentration) for 15 min at room temperature. Then, PDB polymer $(3.33 \mathrm{mg} /$ $\mathrm{mL}$ polymer concentration) was added to the mixture to achieve a final $\mathrm{N}: \mathrm{P}$ ratio of $12: 1$ and incubated for $30 \mathrm{~min}$ at room temperature. Fivefold excess of $10 \mathrm{~mm}$ phosphate buffer ( $\mathrm{pH}$ 8.0) was added to the samples before diluting in fivefold excess of respective growth media. A final siRNA concentration of $100 \mathrm{~nm}$ was used to treat cells.

\section{Statistics}

Data presentation and statistics were generated using GraphPad Prism 6 software. Significance was determined by analysis of variance (ANOVA) with Bonferroni posthoc tests followed by Student's unpaired two-tailed $t$-test for experiments with more than two conditions, or a Student's unpaired two-tailed $t$-test for experiments with only two conditions $(P \geq 0.05)$. For tumor growth curves, significance was determined by area under the curve.

\section{Acknowledgements}

This work was supported by Specialized Program of Research Excellence (SPORE) grant NIH P50 CA098131 (VICC), Cancer Center Support grant NIH P30 CA68485 (VICC), NIH F31 CA195989-01 (MMW) and CTSA UL1TR000445 from the National Center for Advancing Translational Sciences.

\section{Author details}

${ }^{1}$ Department of Cancer Biology, Vanderbilt University School of Medicine, Nashville, TN, USA. ²Department of Biomedical Engineering, Vanderbilt University School of Engineering, Nashville, TN, USA. ${ }^{3}$ Department of Medicine, Vanderbilt University Medical Center, Nashville, TN, USA. ${ }^{4}$ Department of Medicine, Stanford University School of Medicine, Stanford, CA, USA. ${ }^{5}$ Cancer Therapy and Research Center, University of Texas Health Science Center, San Antonio, TX, USA. ${ }^{6}$ The Vanderbilt-Ingram Cancer Center, Vanderbilt University Medical Center, Nashville, TN 37232, USA

\section{Competing interests}

The authors declare that they have no competing financial interests.

\section{Publisher's note}

Springer Nature remains neutral with regard to jurisdictional claims in published maps and institutional affiliations.

Supplementary Information accompanies this paper at https://doi.org/ 10.1038/s41419-017-0072-x.

Received: 13 May 2017 Revised: 10 August 2017 Accepted: 17 August 2017 Published online: 17 January 2018

References

1. American Cancer Society. Cancer Facts \& Figures 2016 (ed. Society, A. C.) (American Cancer Society, Atlanta, 2016).

2. Musgrove, E. A. \& Sutherland, R. L. Biological determinants of endocrine resistance in breast cancer. Nat. Rev. Cancer 9, 631-643 (2009).

3. Massarweh, S. \& Schiff, R. Unraveling the mechanisms of endocrine resistance in breast cancer: new therapeutic opportunities. Clin. Cancer Res. 13, 1950-1954 (2007). 
4. Hanahan, D. \& Weinberg, R. A. The hallmarks of cancer. Cell 100, 57-70 (2000).

5. Tait, S. W. \& Green, D. R. Mitochondria and cell death: outer membrane permeabilization and beyond. Nat. Rev. Mol. Cell Biol. 11, 621-632 (2010).

6. Chipuk, J. E., Bouchier-Hayes, L. \& Green, D. R. Mitochondrial outer membrane permeabilization during apoptosis: the innocent bystander scenario. Cell Death Differ. 13, 1396-1402 (2006).

7. Willis, S. N. et al. Proapoptotic Bak is sequestered by Mcl-1 and BCl-xL, but not BCl-2, until displaced by BH3-only proteins. Genes Dev. 19, 1294-1305 (2005).

8. Cheng, E. H. et al. BCL-2, BCL-X(L) sequester BH3 domain-only molecules preventing BAX- and BAK-mediated mitochondrial apoptosis. Mol. Cell 8, 705-711 (2001).

9. Olopade, O. I. et al. Overexpression of $\mathrm{BCL}-\mathrm{x}$ protein in primary breast cancer is associated with high tumor grade and nodal metastases. Cancer J. Sci. Am. 3, 230-237 (1997).

10. Ding, Q. et al. Myeloid cell leukemia-1 inversely correlates with glycogen synthase kinase-3beta activity and associates with poor prognosis in human breast cancer. Cancer Res. 67, 4564-4571 (2007).

11. Oakes, S. R. et al. Sensitization of BCL-2-expressing breast tumors to chemotherapy by the BH3 mimetic ABT-737. Proc. Natl. Acad. Sci. USA 109, 2766-2771 (2012).

12. Williams, M. M. et al. Key survival factor, Mcl-1, correlates with sensitivity to combined Bcl-2/Bcl-xL blockade. Mol. Cancer Res. 15, 259-268 (2017).

13. Vaillant, F. et al. Targeting BCL-2 with the BH3 mimetic ABT-199 in estrogen receptor-positive breast cancer. Cancer Cell. 24, 120-129 (2013).

14. Thrane, S. et al. A kinase inhibitor screen identifies Mcl-1 and Aurora kinase A as novel treatment targets in antiestrogen-resistant breast cancer cells. Oncogene 34, 4199-4210 (2015).

15. Crawford, A. C., Riggins, R. B., Shajahan, A. N., Zwart, A. \& Clarke, R. Co-inhibition of BCL-W and BCL2 restores antiestrogen sensitivity through BECN1 and promotes an autophagy-associated necrosis. PLOS ONE 5, e8604 (2010).

16. Zhou, H., Zhang, Y., Fu, Y., Chan, L. \& Lee, A. S. Novel mechanism of antiapoptotic function of 78-kDa glucose-regulated protein (GRP78): endocrine resistance factor in breast cancer, through release of B-cell lymphoma 2 (BCL2) from BCL-2-interacting killer (BIK). J. Biol. Chem. 286, 25687-25696 (2011).

17. Williams, M. M. \& Cook, R. S. BCl-2 family proteins in breast development and cancer: could Mcl-1 targeting overcome therapeutic resistance? Oncotarget $\mathbf{6}$ 3519-3530 (2015)

18. Martin, L. A. \& Dowsett, M. BCL-2: a new therapeutic target in estrogen receptor-positive breast cancer? Cancer Cell. 24, 7-9 (2013).

19. Osborne, C. K., Coronado, E. B. \& Robinson, J. P. Human breast cancer in the athymic nude mouse: cytostatic effects of long-term antiestrogen therapy. Eur. J. Cancer Clin. Oncol. 23, 1189-1196 (1987).

20. Oltersdorf, T. et al. An inhibitor of $\mathrm{BCl}-2$ family proteins induces regression of solid tumours. Nature 435, 677-681 (2005).

21. Massarweh, S. et al. Tamoxifen resistance in breast tumors is driven by growth factor receptor signaling with repression of classic estrogen receptor genomic function. Cancer Res. 68, 826-833 (2008).

22. Leverson, J. D. et al. Potent and selective small-molecule MCL-1 inhibitors demonstrate on-target cancer cell killing activity as single agents and in combination with ABT-263 (navitoclax). Cell Death Dis. 6, e1590 (2015).

23. Kotschy, A. et al. The MCL1 inhibitor $\mathbf{S 6 3 8 4 5}$ is tolerable and effective in diverse cancer models. Nature 538, 477-482 (2016).

24. Lee, T. et al. Discovery and biological characterization of potent myeloid cell leukemia-1 inhibitors. FEBS Lett. 591, 240-251 (2017).

25. AMG 176 first in human trial in subjects with relapsed or refractory multiple myeloma [Internet], 2015. Available from: https:/clinicaltrials.gov/ct2/show/ NCT02675452. Accessed 13 July 2017.

26. Miller, T. W. et al. Hyperactivation of phosphatidylinositol-3 kinase promotes escape from hormone dependence in estrogen receptor-positive human breast cancer. J. Clin. Invest. 120, 2406-2413 (2010).

27. Tse, C. et al. ABT-263: a potent and orally bioavailable BCl-2 family inhibitor. Cancer Res. 68, 3421-3428 (2008).

28. Wang, B. et al. The BCl-2/XL inhibitor ABT-263 increases the stability of MCl-1 mRNA and protein in hepatocellular carcinoma cells. Mol. Cancer 13, 98 (2014).

29. Pan, R. et al. Inhibition of Mcl-1 with the pan-Bcl-2 family inhibitor (-)Bl97D6 overcomes ABT-737 resistance in acute myeloid leukemia. Blood 126, 363-372 (2015).

30. Faber, A. C. et al. Assessment of ABT-263 activity across a cancer cell line collection leads to a potent combination therapy for small-cell lung cancer. Proc. Natl. Acad. Sci. USA 112, E1288-E1296 (2015).
31. Faber, A. C. et al. mTOR inhibition specifically sensitizes colorectal cancers with KRAS or BRAF mutations to BCL-2/BCL-XL inhibition by suppressing MCL-1. Cancer Discov. 4, 42-52 (2014).

32. Anderson, G. R. et al. PIK3CA mutations enable targeting of a breast tumor dependency through mTOR-mediated MCL-1 translation. Sci. Transl. Med. 8, $369 r a 175$ (2016).

33. Hur, J. et al. Regulation of expression of BIK proapoptotic protein in human breast cancer cells: p53-dependent induction of BIK mRNA by fulvestrant and proteasomal degradation of BIK protein. Cancer Res. 66, 10153-10161 (2006).

34. Hur, J. et al. The Bik BH3-only protein is induced in estrogen-starved and antiestrogen-exposed breast cancer cells and provokes apoptosis. Proc. Natl. Acad. Sci. USA 101, 2351-2356 (2004).

35. Gullberg M. A.C. A. Visualization and quantification of protein-protein interactions in cells and tissues. Nat. Methods $\mathbf{7}(2010)$.

36. Werfel T. A. et al. Combinatorial optimization of PEG architecture and hydrophobic content improves siRNA polyplex stability, pharmacokinetics, and potency in vivo. J. Control Release 255, 12-16 (2017)

37. Massarweh, S. et al. A phase II neoadjuvant trial of anastrozole, fulvestrant, and gefitinib in patients with newly diagnosed estrogen receptor positive breast cancer. Breast Cancer Res. Treat. 129, 819-827 (2011).

38. Samarnthai, $\mathrm{N}$. et al. Pathologic changes in breast cancer after anti-estrogen therapy. Breast J. 18, 362-366 (2012).

39. Pink, J. J., Bilimoria, M. M., Assikis, J. \& Jordan, V. C. Irreversible loss of the oestrogen receptor in T47D breast cancer cells following prolonged oestrogen deprivation. Br. J. Cancer 74, 1227-1236 (1996).

40. Crawford, A. \& Nahta, R. Targeting BCl-2 in herceptin-resistant breast cancer Cell lines. Curr. Pharmacogenomics Person. Med. 9, 184-190 (2011).

41. Balko, J. M. et al. Molecular profiling of the residual disease of triple-negative breast cancers after neoadjuvant chemotherapy identifies actionable therapeutic targets. Cancer Discov. 4, 232-245 (2014).

42. Real, P. J. et al. Resistance to chemotherapy via Stat3-dependent overexpression of Bcl-2 in metastatic breast cancer cells. Oncogene 21, 7611-7618 (2002).

43. Teixeira, C., Reed, J. C. \& Pratt, M. A. Estrogen promotes chemotherapeutic drug resistance by a mechanism involving $\mathrm{BCl}-2$ proto-oncogene expression in human breast cancer cells. Cancer Res. 55, 3902-3907 (1995).

44. A. Phase $1 B$ study of $B C-2$ inhibition with $A B T-299$ in combination with tamoxifen in metastatic ER-positive breast cancer [Internet], 2015. Available from: https://www.anzctr.org.au/Trial/Registration/TrialReview.aspx?id=368782. Accessed 3 June 2017

45. Joensuu, H., Pylkkanen, L. \& Toikkanen, S. BCl-2 protein expression and longterm survival in breast cancer. Am. J. Pathol. 145, 1191-1198 (1994).

46. Williams $\mathrm{M}$. et al. Key sunvival factor, $\mathrm{Mcl}-1$, correlates with sensitivity to combined BCl-2/BCl-xL blockade. Mol. Cancer Res. (2016).

47. Schacter, J. L., Henson, E. S. \& Gibson, S. B. Estrogen regulation of antiapoptotic BCl-2 family member Mcl-1 expression in breast cancer cells. PLoS ONE 9, e100364 (2014).

48. Xiao, Y. et al. MCL-1 is a key determinant of breast cancer cell survival: validation of MCL-1 dependency utilizing a highly selective small molecule inhibitor. Mol. Cancer Ther. 14, 1837-1847 (2015).

49. Roberts, A. W. et al. Substantial susceptibility of chronic lymphocytic leukemia to BCL2 inhibition: results of a phase I study of navitoclax in patients with relapsed or refractory disease. J. Clin. Oncol. 30, 488-496 (2012).

50. Wilson, W. H. et al. Navitoclax, a targeted high-affinity inhibitor of BCL-2, in lymphoid malignancies: a phase 1 dose-escalation study of safety, pharmacokinetics, pharmacodynamics, and antitumour activity. Lancet Oncol. 11, 1149-1159 (2010).

51. Wang, $X$. et al. Deletion of MCL-1 causes lethal cardiac failure and mitochondrial dysfunction. Genes Dev. 27, 1351-1364 (2013).

52. Arbour, N. et al. MCl-1 is a key regulator of apoptosis during CNS development and after DNA damage. J. Neurosci. 28, 6068-6078 (2008).

53. Hasan, S. M. et al. Mcl1 regulates the terminal mitosis of neural precursor cells in the mammalian brain through p27Kip1. Development 140, 3118-3127 (2013).

54. Peperzak, V. et al. Mcl-1 is essential for the survival of plasma cells. Nat. Immunol. 14, 290-297 (2013).

55. Dzhagalov, I., Dunkle, A. \& He, Y. W. The anti-apoptotic BCl-2 family member Mcl-1 promotes T lymphocyte survival at multiple stages. J. Immunol. 181, 521-528 (2008). 
56. Dzhagalov, I., St John, A. \& He, Y. W. The antiapoptotic protein Mcl-1 is essential for the survival of neutrophils but not macrophages. Blood 109 1620-1626 (2007).

57. Iyer, A. K., Khaled, G., Fang, J. \& Maeda, H. Exploiting the enhanced permeability and retention effect for tumor targeting. Drug Discov. Today 11, 812-818 (2006).
58. Singh, R. \& Lillard, J. W. Jr. Nanoparticle-based targeted drug delivery. Exp. Mol. Pathol. 86, 215-223 (2009).

59. Anselmo, A. \& Mitragotri, S. Nanoparticles in the clinic. Bioeng. Transl. Med. 1, 10-29 (2016).

60. Morrison, M. M. et al. ErbB3 downregulation enhances luminal breast tumor response to antiestrogens. J. Clin. Invest. 123, 4329-4343 (2013). 